\title{
Reducing avoidable time delays in immediate medication administration - learning from a failed intervention
}

Sachin Nagar, nicola davey

University Hospitals Leicester NHS Trust

\begin{abstract}
Stat medications are regularly prescribed on hospital wards as part of the ongoing care for patients. Because they are prescribed at variable times that do not coincide with regular nursing drug administration times, they rely on good communication and vigilance on staff to ensure they are administered in a timely manner. Delays in drug administration can lengthen patient recovery times, prolong admission, and can lead to avoidable patient harm and suffering.
\end{abstract}

While working on a geriatrics ward I noticed that there were often significant delays in administration of stat medications which occurred on a regular basis. I therefore investigated this by collecting data over a two week period to assess the situation based on our current practice. After root cause analysis (figure 1), it became clear that communication between staff was a significant factor in delayed administration. A solution was implemented in the form of "ward bay wall charts" to aid documentation and communication of stat medication requirements between nursing and medical staff with the intention to reduce delays by improving communication.

After gaining support of medical and nursing staff, a trial was undertaken and a further two weeks of data collected to see the effect of the intervention. The results showed that there was an increase in the median time delay (1 hour 34 minutes to 2 hours 26 minutes, a 55\% increase in median time delay) after the implementation of the my intervention, suggesting that it actually made communication worse, creating more delays. Subsequent feedback and analysis showed that this was due to a number of factors that led to worsened communication between staff and therefore an increase in medication delays. Early recognition allowed the intervention to be promptly withdrawn and a reassessment of the nature of the initial problem.

This project highlights the importance of measurement in determining if an intervention actually works and is an improvement on current practice.

\section{Problem}

Whenever patients are reviewed by medical staff it invariably leads to changes in management and the prescription of stat medications to try to rapidly bring about a change in a patient's clinical condition. For this to be effectively converted from a medical plan to an administered drug, a number of steps need to occur in a timely manner:

1. The drug first need to be prescribed

2. Be available on the ward

3. Be communicated to nursing staff

4. Be administered via the route prescribed. At each point in this chain of events there is the possibility of delay.

A subjective review of the practice on ward 19 at the Leicester Royal Infirmary (UK) (30 bed ward) revealed that often some stat medications were being significantly delayed, leading to subsequent delays in improvement of patient symptoms, ie administration of laxatives and subsequent resolution of constipation. The effect of this was prolonged stay on the medical ward, slowing discharge and increasing the risk of hospital acquired infections. A major factor identified by staff was a perceived lack of communication between medical and nursing staff, leading to a delay in awareness of the need for administration. This communication problem was compounded by the fact that the nursing staff were too short staffed to join the medical ward rounds, leading to ad-hoc handovers throughout the day.

\section{Background}

Delays in medication administration have been identified by the National Patient Safety Agency (NPSA) as being a significant patient safety issue, as documented in their Rapid Response Alert $1183 \mathrm{~A}$. One example of avoidable harm resulting from medication administration delay documented in the report, included a case of delayed antimicrobial administration. A patient with cellulitis did not receive timely antibiotics due to delayed administration, leading her to become severely septic and subsequently die.[1]

The Parkinson's Disease Society have also reported concerns regarding avoidable delays in medication administration in their "Get it on time" campaign, highlighting in particular the detrimental effect of delaying anti-Parkinson's medication on the recovery of patients with Parkinson's disease.[2] The potential for harm depends on the clinical scenario and the particular medication in question. As such, certain medications have been identified as being time crucial and include insulin, anti-infectives, and 
BMJ Quality Improvement Reports

anticoagulants.[1] However, all prolonged medication delays could reasonably be considered as detrimental to patient recovery. In the case of pre-renal acute kidney injury, it is well known that early identification and treatment with fluid resuscitation leads to reduced morbidity [3] and therefore shorter hospital admissions. As such it is important to try to minimise unnecessary delays by finding workable solutions to improving communication and timely medication administration.

There have been reports of successful interventions to address harm caused by medication delays, by using specific features within electronic prescribing systems to reduce the impact of human error. These have included features within the programs to either remind staff of pending drugs by highlighting them on a "clinical dashboard," [4] or by automatically adjusting antibiotic administrations times to ensure they are given within a minimum time frame from time of prescription.[5]

\section{Baseline measurement}

The baseline measurement was performed prospectively by reviewing the electronic drug charts of all the patients on ward 19 at the Leicester Royal Infirmary Hospital over a two week period. The number of and type of stat medications prescribed during this two week period were identified as the baseline data source. Each medication was then reviewed to find the time of prescription and subsequent administration, so that the time delay could be calculated and recorded in a spreadsheet. This data was used to create a run chart to view our current practice and assess the scale of the problem (figure 2). Medications prescribed or administered outside this time period were excluded as were medications prescribed prior to patients being transferred to ward 19. The latter prescriptions were identified by reviewing the medical notes and comparing ward admission times with drug prescription times.

During the two week period a total of 99 stat medications were prescribed and administered on ward 19 . The results showed that there was a significant variability in the time delay between medications being prescribed and given. The median time delay was ( 1 hour 34 minutes) and formed the means by which the intervention success would be judged (lower quartile $=26$ minutes, upper quartile $=4$ hours 46 minutes, maximum $=24$ hours, minimum $=0$ minutes .

See supplementary file: ds4608.png - "Fishbone diagram illustrating root cause analysis of factors affecting timely stat medication administration"

\section{Design}

A root cause analysis of the the potential barriers to the timely administration of stat medications identified a number of factors that could contribute to delays. Communication between medical and nursing staff regarding when a medication was prescribed was found to be a major contributory factor to significant delays. A solution was sought to address this issue by finding a simple way to aid communication between the two staffing groups as to when stat medications were prescribed.

The idea for having a "ward bay wall chart" was developed from a similar system being used successfully on the acute medical unit (AMU) at the Leicester Royal Infirmary. White boards at the entrance to each bay showed each bed in the bay and in adjacent side rooms. Pending tasks such as urine samples or enema administrations were recorded by nursing staff to serve as both a communication tool and a memory aid.

I adapted this idea on ward 19 as a communication aid between medical and nursing staff by creating simple laminated wall charts that could be attached to the entrance of the bays. The rational was that medical staff would prescribe stat medications on the drug chart then inform the nurse verbally of the prescription. The message would then also be written on the laminated white charts as a reminder for both medical and nursing staff of stat medications outstanding. The message would be wiped off once the medication was administered. The intention being that if the message was not wiped off, it would prompt the nurse or doctor to investigate further the cause of the delay. A3 Laminated charts were chosen for the trial period as they were significantly cheaper and easier to put in place as a temporary intervention, than dry wipe white boards. The aim was that if they were a successful addition, that they could be replaced with actual white boards for use on a permanent basis

Staff were informed of the quality improvement project aims and also asked for feedback and suggestions on the proposed intervention to address the issue of medication delays. After discussion with nursing and medical staff on the ward, the design was altered to ensure anonymity of individual patients by avoiding the use of names. It also defined what information would be written on the charts to ensure effective communication while maintaining patient confidentiality. The latter was achieved by using abbreviations to simply indicate the type of stat medication prescribed and the time of prescription.

Before implementation there was a feeling among some staff that the intervention may not work as well as hoped due to a perceived increase in workload created by the wall charts. This was discussed and an agreement was reached to simply try the intervention with the view to removing it should it prove to be too onerous or unworkable as a solution.

\section{Strategy}

PDSA cycle 1: The first task was to design and create the wall charts. A design for the wall charts was produced and reviewed by medical and nursing staff on the ward so that changes could be made to the template prior to printing. Changes were made to the design using this feedback, which was then taken to a local printing shop for printing and lamination. The posters were put at the entrance of each bay ready for the two week trial. The intervention was explained to all staff on the understanding that it was to be used in conjunction with verbal communication between staff as a two week trial.

At the start of the trial it was found that the dry wipe makers would 
not wipe off easily from the laminated surface of the charts if allowed to dry for a long period of time. This hindered the usage of the charts as nursing staff were reluctant to try to wipe the messages off due to this. A simple solution was suggested by one of the nurses to use a small amount of alcohol foam from the hand sanitisers to wipe off the messages. This temporary work-around was trialled and worked very well, so was then disseminated between the staff to help assist them during the remainder of the two week trial.

PDSA cycle 2: This was to assess how well the intervention would work in the future. To assess this the final four days of the two week trial were used to see how the lack of my presence on the ward would affect both the use of the intervention and the subsequent time delays in administering stat medications. I was timetabled to be away from the ward so used this as an opportunity to collect data on my return to see if the intervention would be sustainable if the results showed it had been successful.

\section{Results}

Prescription and administration times were collected by reviewing the electronic drug charts over a two week period following the implementation of the wall charts on the ward. A total of 110 stat medications were prescribed in this period, 28 of which were prescribed on the final four days while I was away for the ward.

The effect of the intervention was a $55 \%$ increase in the median time delay for stat medication administration (median delay: 1 hour 34 minutes pre-intervention vs 2 hour 26 minutes post intervention) (figure 2). The variability of delay was also greater after the intervention, (pre-intervention: lower quartile $=26$ minutes, upper quartile $=4$ hours 46 minutes, vs post intervention: lower quartile $=$ 58 minutes, upper quartile $=7$ hours 5 minutes) indicating that the initial intervention had actually caused a worsening of timely stat medication administration. Subsequent analysis of the final four days of the post intervention data collection cycle, demonstrated an even greater increase in both the median time delay ( 2 hours 52 minutes, a $83 \%$ increase from the pre intervention median) and the variability of the delays in this period (lower quartile $=1$ hour 07 minutes, upper quartile $=9$ hours 15 minutes). The result indicating that my presence off the ward at the end of the data collecting cycle further increased the time delay in stat medication administration.

See supplementary file: ds4609.png - "Run chart showing the change in median stat medication administration delay pre and post intervention"

\section{Lessons and limitations}

After reflecting on the unsuccessful outcome of the intervention, a number of important learning points became apparent. From a positive point of view, the intervention chosen was simple and cheap to produce. The four white boards in total cost around $£ 30$ and allowed for easy implementation. Data collection was facilitated by using the electronic prescribing software being used on the ward, allowing for quick and easy recording of prescription and drug administration times from a single computer terminal. It also allowed frequent data collection over a short period of time, providing ample data with which to analyse changes in administration delays.

However, there were some notable problems encountered during the project that now serve as valuable learning points for future projects. First of all was the fact that the intervention increased the workload of staff and added an extra step in the standard operating procedure for stat medication administration. This was perhaps a fundamental reason why the intervention did not catch on or be used as intended. Along with this was the fact that the white boards themselves could be easily circumvented by staff. They could ignore them and continue as "normal" without any added difficulty, which some of them did.

Alternatively some staff used it as a reason not to communicate verbally, worsening inter-professional communication and subsequently causing the increase in delays observed. It is not possible to comment on whether the intervention would have been sustainable as it was not successful. However, the worsening of the delays at the end of the study period indicate it was unlikely to be sustainable even if it had produced a reduction in time delays. The final increase in delay was likely due to a lack of my presence on the ward enforcing use of the white boards, ie the intervention relied on vigilance and insufficiently addressed the need for a system change.

The idea for the intervention originated from a similar tool that was seen used successfully on the AMU. It was assumed that a similar intervention would work well on ward 19, although this did not turn out to be the case. Looking back more closely at the system used on the AMU, it became clear that the white boards there were being used by nursing staff to simply keep track of their jobs, ie it was for communication between nurses working in a single bay rather than between different professional groups. This subtlety likely played a role in the failure of my intervention as I was attempting to use it for communication between professionals that have differing agendas.

There were some limitations that may have adversely affected the results and therefore the success of the intervention. As there was no formal teaching session on the use of the white boards it is possible that some staff (particularly night staff and bank staff) may not have been aware of how to use the intervention properly. An attempt was made to address this by speaking to staff directly, however in retrospect a formal teaching session with written information or follow up email may have improved this further. There was also the potential for systematic error, particularly when it came to intravenous fluid prescription as often multiple bags would be prescribed at the same time. This would mean some bags of fluid would appear to be significantly delayed when in reality they were put up immediately after the previous bag had been completed. To some extent this error was mitigated by using the median time delay to observe the change in delayed administration, thereby excluding the extreme outliers.

\section{Conclusion}

Despite the unsuccessful implementation of the intervention this 
project did highlight the importance of collecting accurate data with which to assess an intervention. Because of this it was easy to see that the intervention had not been successful, allowing it to be withdrawn early instead of persisting to the detriment of patients. The lessons learned from this project were valuable and will serve as a reminder of important factors to consider when designing a solution to improve quality in the healthcare setting. With regards to reducing delays in stat medication, my next step would be to perhaps focus solely on investigating and improving delays in critical medications rather than all medications as this may be more clinically relevant and more manageable to improve.

\section{References}

1. National Patient safety Agency. Reducing harm from omitted and delayed medicines in hospital. 2010. http://www.nrls.npsa.nhs.uk/alerts/?entryid45 $=66720$

2. Parkinson's Disease Society. Get It On Time campaign. 2014 http://www.parkinsons.org.uk/content/get-it-timecampaign-what-hospitals-can-do

3. Joslin J. Ostermann M. Care of the Critically III Emergency Department Patient with Acute Kidney Injury. Emergency Medicine International Print. 2012:760623, 2012. http://www.hindawi.com/journals/emi/2012/760623/

4. Coleman JJ, Hodson J, Brooks HL, Rosser D. Missed medication doses in hospitalised patients: a descriptive account of quality improvement measures and time series analysis. Int J Qual Health Care. Oct 2013; 25(5): 564-72. http://www.ncbi.nlm.nih.gov/pmc/articles/PMC3786625/

5. Matthews PC, Wangrangsimakul T, Borthwick M, Williams C, Byren I, Wilkinson D. Electronic prescribing: Reducing delay to first dose of antibiotics for patients in intensive care. BMJ Qual Improv Report 2013;2: doi:10.1136/bmjquality.u202241.w1120. http://qir.bmj.com/content/2/2/u202241.w1120.full

\section{Declaration of interests}

Nothing to declare.

\section{Acknowledgements}

This project was completed within the BaSIS programme for doctors in training run by Health Education East Midlands.

Additional support and coaching was provided by Nicola Davey, quality improvement practitioner, Quality Improvement Clinic Ltd. 